\title{
Underwater Pulse-Current FCAW - Part 2: Bubble Behaviors and Waveform Optimization
}

\author{
Droplet detachment was facilitated by controlling the bubble separation modes
}

\author{
BY J. WU, Y. HAN, C. JIA, C. WU, AND Q. YANG
}

\begin{abstract}
Underwater pulse-current wet welding was proposed in part 1 of this two-part report. The novel technology obtained improved metal transfer and welding process stability. The main reason for droplet oversizing and long transfer cycles was found to be the deviated large droplet stage. In this part, the waveform optimization for both bubble behaviors and metal transfer were investigated. Efforts were made for shortening the duration of the deviated large droplet stage. Pulse current influences on bubble evolution was studied. It was found that two different separation modes can be adjusted by appropriately changing the current values when the bubbles are necking. Quickly reducing the welding current can sharply lower the impact force on the droplets due to intense gas flow changes inside. Under the optimized pulse current, the range of the metal transfer cycle became narrower, and droplet diameters were smaller than that of the original condition. Stable and improved metal transfer processes were achieved with a frequency of $7.52 \mathrm{~Hz}$ and an average droplet diameter of $2.4 \mathrm{~mm}$, which was about 1.5 times the wire diameter. The optimized pulse waveform greatly improved weld formation with less spatter and a more uniform appearance.
\end{abstract}

\section{KEYWORDS}

- Underwater Welding • Metal Transfer • Bubble Evolution - Pulse Current • Process Control

\section{Introduction}

In underwater wet flux cored arc welding (FCAW), the unstable metal transfer and generated spatter deteriorate process stability and weld quality. The metal transfer process has characteristics such as oversize, low frequency, unregulated trajectory, etc. Based on visual sensing tech- nologies, it is thought that the main metal transfer mode is large droplet repel transfer (Refs. 1, 2). Typically, the droplet size is about four times the wire diameter, the metal transfer cycle is about $0.225 \mathrm{~s}$, and the transfer frequency is about $4.44 \mathrm{~Hz}$. The slow-moving, large droplets are unstably transferred to the weld pool. As a result, there is an intense impact on the weld pool along with bubble disturbances on the transferring droplets, producing spatter and a bad weld appearance.

In part 1 of this two-part report, a novel underwater pulse-current FCAW process was proposed to periodically change the forces applied on droplets (Ref. 3). The regulated metal transfer processes had the maximum droplet diameter of less than $5 \mathrm{~mm}$; large droplet repel transfer was achieved without hardly any unstable short-circuit transfer and surface tension transfer; and the performance of the pulse-current FCAW was much better than the constant-voltage condition. According to the divided six metal transfer stages, the long duration of the deviated large droplet stage was the main factor deciding the long transfer cycle. Under the present pulse current, the time length of the deviated large droplet stage was successfully decreased but with a wide range (30-200 ms). The pulse-current effect was still not stable and needs further optimization.

According to previous research, the bubbles' behaviors are critical for maintaining stable arc burning and smooth metal transfer (Ref. 4). The spatter generation processes are closely related to the bubble evolution (Ref. 5). In part 1 of this two-part report, the related bubble behaviors in pulsecurrent welding and the relevant influences were neglected.

In part 2 of this two-part report, cooperative regulation of both bubble evolution and metal transfer was investigated. The specific effect of bubble necking on metal transfer were revealed. By exploring the influencing mechanisms of pulse current on wet FCAW, the pulse-current waveform was optimized for higher-frequency, smaller-size metal transfer in a stable welding process. 


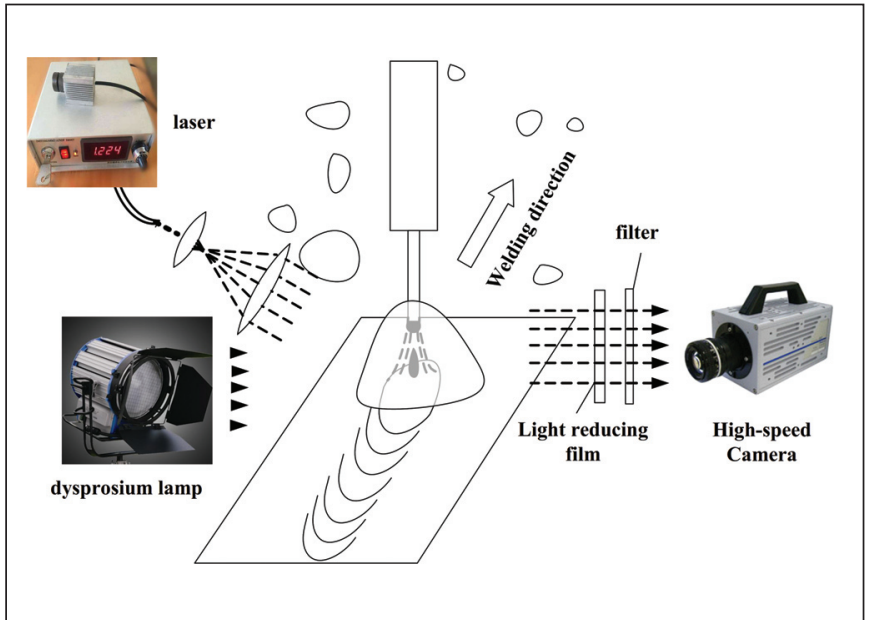

Fig. 1-Equipment platform and signal acquisition system.

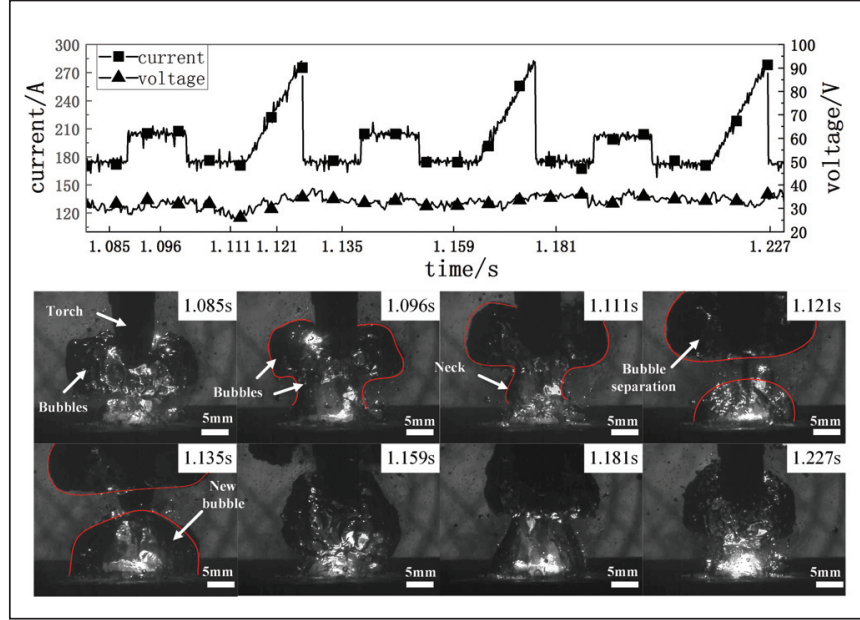

Fig. 2 - Bubble behaviors under a $20-\mathrm{Hz}$ pulse current.

Table 1 - Experiment Parameters with Pulse Current

\begin{tabular}{|c|c|c|c|c|}
\hline Welding Parameters & Wire Feed Rate & Welding Speed & Wire Diameter & Average Current \\
\hline Values & $4.4 \mathrm{~m} / \mathrm{min}$ & $180 \mathrm{~mm} / \mathrm{min}$ & $1.6 \mathrm{~mm}$ & $200 \mathrm{~A}$ \\
\hline
\end{tabular}

\section{Experimental Procedure}

To obtain clear bubble images, a dysprosium lamp was employed as the additional background light source on the basis of the previously developed experimental platform (Ref. 1). As shown in Fig. 1, the dysprosium lamp can provide a large-diameter, ultra-bright background $(0.5 \mathrm{~m})$, which is a great supplement to the laser beam source for observing the bubble behaviors. Bead-on-plate welding experiments were carried out under the water depth of $0.5 \mathrm{~m}$. The base metal was Q235 with a thickness of $8 \mathrm{~mm}$. The consumable was a self-shielded, rutile-type, flux-cored wire (PPS-AN1, $1.6 \mathrm{~mm}$ diameter), which is developed by the E. O. Paton Electric Welding Institute, Kiev, Ukraine, especially for underwater wet welding. The electrode extension was 16 $\mathrm{mm}$. The torch and wire were directly positioned in a water tank. Most of the experimental procedures were the same as those in part 1 of this two-part report. Experiments were first conducted using the existing original pulse-current waveform. The related experimental parameters are shown in Table 1. Based on the acquired images, the bubble behaviors were visually investigated to evaluate the pulse-current influences.

\section{Results}

\section{Effect of Pulse Current on Bubble Behaviors}

The change of current can influence the behavior of bubbles. Therefore, relevant experiments were carried out for the purpose of regulating and controlling the growth behavior of bubbles using pulse current. The pulse-current waveform used in the experiment was unchanged, and the values of each section were adjusted. The current-output frequency was changed from 60 to $20 \mathrm{~Hz}$, i.e., the cycle of each waveform was adjusted from 16.7 to 50 ms. Reducing the frequency meant reducing the frequency of current variation at the same time. By reducing the degree of arc variation, the bubbles were more regular and no longer fragmented, so they could be regulated to a certain extent.

Based on the relationship between bubble formation frequency and average current levels, the output pulse frequency was set accordingly. For example, when the average current was $190 \mathrm{~A}$, the frequency of bubble formation was 18 $\mathrm{Hz}$. The collected data was randomly selected for observation and analysis. When the output frequency of the pulse current was $20 \mathrm{~Hz}$, the bubble growth behavior was more regular.

Figure 2 shows the synchronously collected electrical and visual signals. A certain relationship between bubble behavior and pulse current could be observed. When the pulse current changed from peak value to base value at 1.096-1.111 s, the instantaneous decrease of the current made the heat production decrease and the arc fluctuate, which led to the breakdown of the internal dynamic balance and the changing bubble shapes. At $1.111 \mathrm{~s}$, there was a neck between the two bubbles. When the length of the neck reached a certain degree, the two bubbles separated and a new bubble below continued to grow.

According to the statistics, the bubble separation behavior during stable welding processes can be generally divided into two modes: complete separation and adhesion separation, as shown in Fig. 3. To more accurately identify the outline of the bubble, it was marked with lines. The left picture shows the complete separation mode of two bubbles, and the right picture shows the bubble adhesion mode in which the two bubbles were not completely separated.

For the bubble complete separation mode, during the for- 


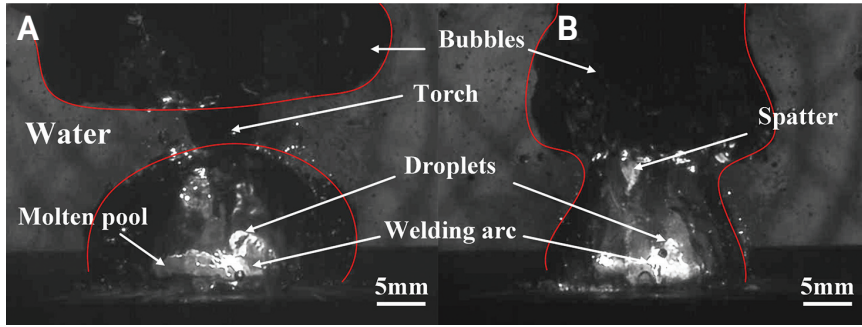

Fig. 3 - Two separation modes of bubbles: A - Complete separation; $B$ - adhesion separation.

mation and growth of the next bubble, the former bubble completely detached from the old bubble, and the two bubbles were not connected, or very few parts were connected. The effect of water pressure on the top of the new bubble was obvious. The main reason for this mode was because, when the bubble separated, the current was low and in the base current section. The volume of gas produced by the decomposition of the core and the arc heat was significantly reduced. As a result, the next bubble was not large enough to adhere to the former bubble when separating, as shown in Fig. 2 (1.121 s). When the necking of the bubble was about to break away at $1.085 \mathrm{~s}$, the current increased to the background current at $1.096 \mathrm{~s}$, which made two bubbles completely unable to separate at $1.096 \mathrm{~s}$. Then, when the current decreased again at $1.121 \mathrm{~s}$, the gas production was insufficient to sustain the growth of the new bubble below to be large enough, so the new bubble directly broke down.

For the bubble adhesion separation mode, the separating old bubble was connected to the newly generated bubble. Both of the two bubbles were sleeve shaped. The main reason for this mode was because the current was still large when the bubble necked and tended to separate. Additionally, the gas production did not decrease, resulting in the expansion of a new bubble directly from the neck of the former bubble. When the former bubble floated up, the new bubble grew and formed a sleeve shape, as shown in Fig. 2, from 1.159 to $1.181 \mathrm{~s}$. Because of the continuous increase of current and the sufficient gas production in this section, the new bubble began to expand directly at the neck of the former bubble when necking occurred.

\section{Pulse-Current Waveform Optimization}

Based on the bubble separation time when the average current was $180 \mathrm{~A}$, the whole waveform cycle was set as 71 ms. Under the pulse-current condition, the bubble separation time was affected by the valley current of the waveform. Considering the delay time of the bubble behaviors, it was extended by $11 \mathrm{~ms}$ in the previous design. In this case, the output-current pulse frequency was $14 \mathrm{~Hz}$.

As shown in Fig. 4, the optimized waveform was designed with four stages. In actual welding experiments, it was found that the time required for droplet growth fluctuated greatly and varied with the change of current levels. According to the previous analysis of the metal transfer process in part 1 of this report, the time for droplet formation (i.e., initial stage, growing up, and deviation) was about 60-70 ms, and the time for the deviated large droplet stage was about 40-50 ms (Ref. 3). It was found that under the same average

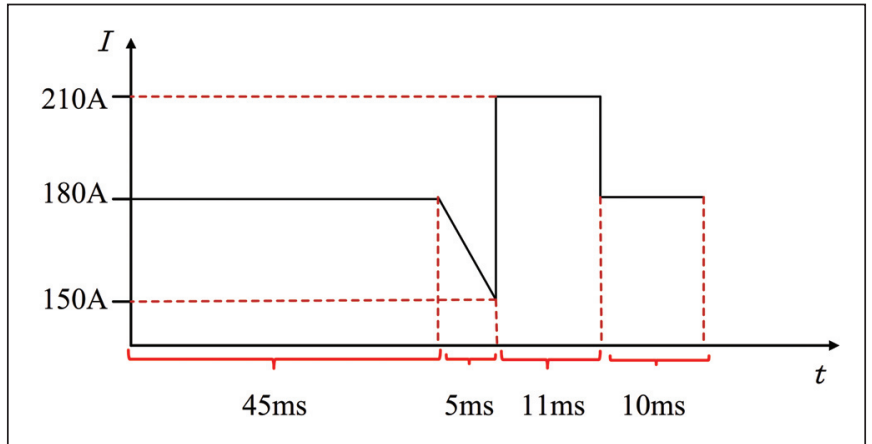

Fig. 4 - Optimized pulse-current waveform in wet FCAW.

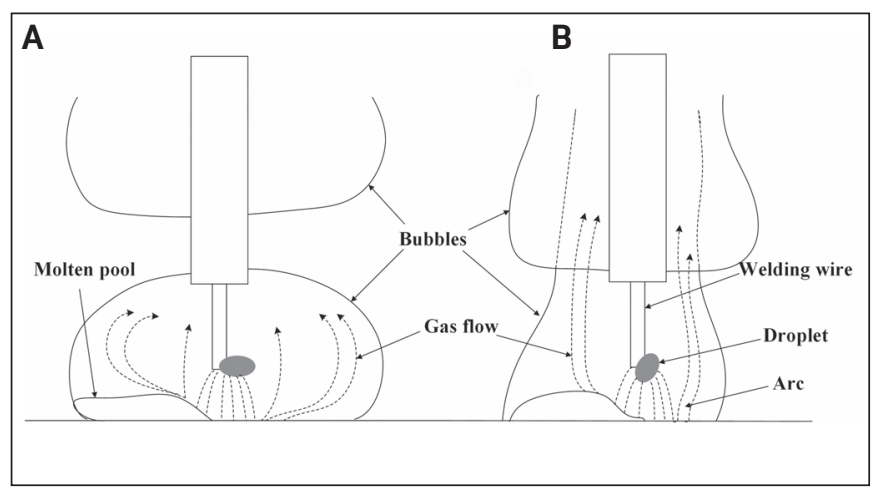

Fig. 5 - Schematic of different gas flows under two separation modes of bubbles: $A-$ Complete separation; $B-$ adhesion separation.

current level, the droplet formation stage took almost the same length of time in the conventional constant-voltage and the pulse-current FCAW processes. Therefore, the purpose of waveform optimization was to significantly reduce the time length of the deviated large droplet stage.

The first section of the waveform ( $45 \mathrm{~ms}$ ) was used to cover the droplet formation stages. A suitable welding current value (210 A) was used to provide sufficient power support for melting the wire quickly and stably. In the second section (following $5 \mathrm{~ms}$ ), the droplets fluctuated when the current decreased and then moved into the deviated large droplet stage ahead of time. At the same time, because of the sudden change of arc burning status, the bubbles necked in advance. The current rose steeply in the third section (11 $\mathrm{ms}$ ) with the variation amplitude of $60 \mathrm{~A}$.

The purpose was to make the droplets deviate immediately and begin to detach from the wire. It is expected that the duration of the deviated large droplet stage can be shortened significantly, or even directly enter the detaching stage. Considering the corresponding bubble behaviors at this stage, the bubble necking tended to accelerate and gradually break away. The previous research verified that increasing the current when the bubble necked produced the complete separation mode. In this way, the quickly separated bubbles could avoid the intense gas flow disturbances on the detaching droplet, and spatter would not be formed without excessive impact and dragging force.

The fourth section of the waveform was designed for droplet transferring, new droplets, and the new bubble formation stage. Because there was an overlap time between 


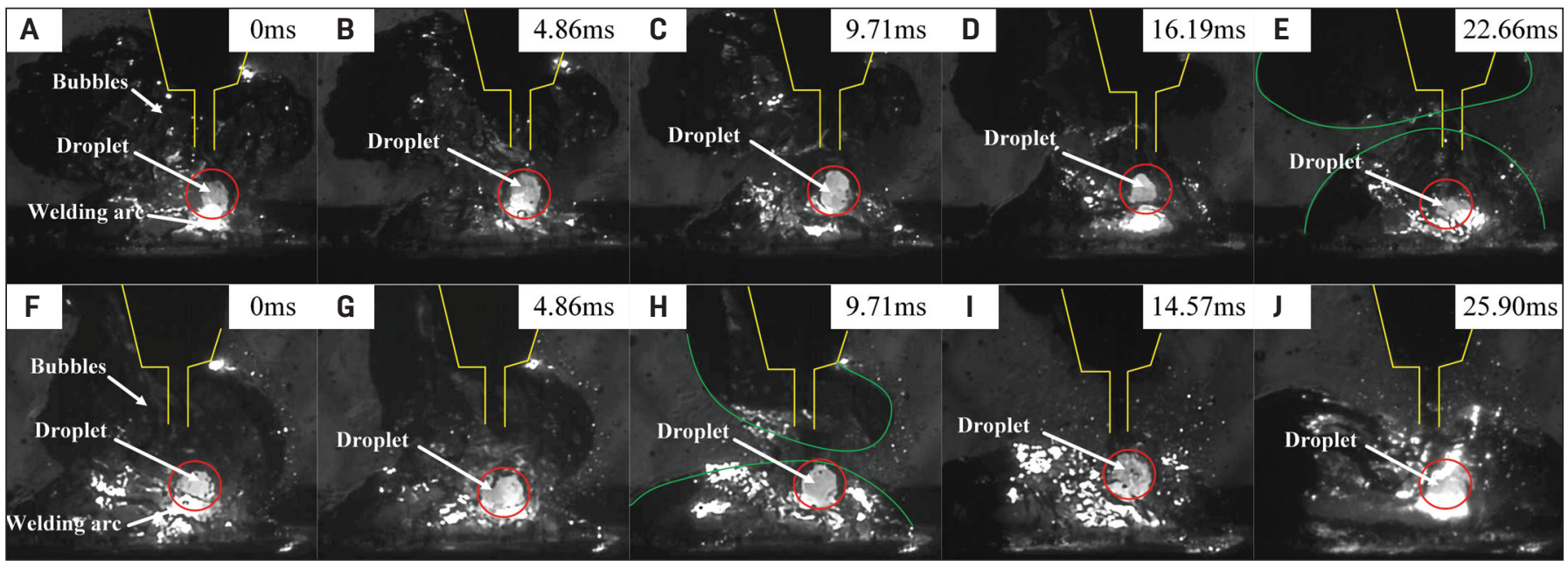

Fig. 6 - Droplet and bubble behaviors in two typical periods under the complete bubble separation mode.

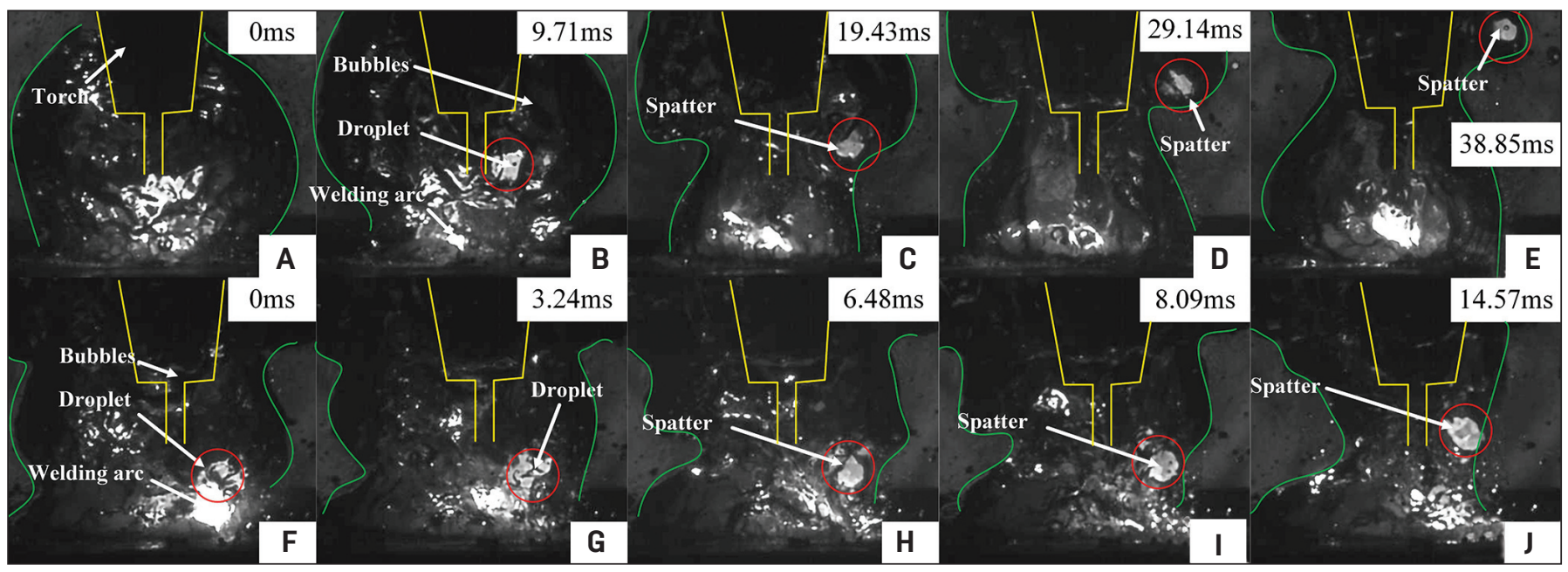

Fig. 7 - Typical spatter formation processes under the adhesion separation mode of bubbles.

the bubble detachment and the growth of new bubbles, there was also a period for droplets to detach from the wire and transfer to the molten pool. Compared with the actual processes, the time from bubble shrinkage to leaving the arc region was approximately $11.3 \mathrm{~ms}$. The complete separation time of bubbles was about $17.8 \mathrm{~ms}$. Because the third and fourth sections of the waveform were used to cover the whole stage from necking to complete separation of bubbles, the fourth section time length of the pulse-current waveform was set as $10 \mathrm{~ms}$. The waveform was used as the preset waveform 1 . The welding parameters were as follows: the arc voltage was $27 \mathrm{~V}$, the wire feed rate was $4.4 \mathrm{~m} / \mathrm{min}$, and the welding speed was $180 \mathrm{~mm} / \mathrm{min}$.

\section{Discussions}

\section{Bubble Evolution Influences on Droplets}

During underwater pulse-current wet FCAW, the different bubble shapes under the two separation modes led to different dragging forces acting on droplets. The bubbles, under the complete separation mode, completely separated from the former bubble. They were ellipsoidal or invertedbowl shaped under the action of water pressure. At this time, the internal gas flow was roughly shown in Fig. 5A. Under the influence of water pressure and water resistance for bubble moving, the bubbles tended to expand horizontally. The acting forces applied on droplets were mainly caused by the gas flow inside the bubbles.

The gas flow was expressed in a dotted line, as shown in Fig. 5A. Please note the gas produced by flux thermal decomposition struck intensely around the end of the welding wire. The distance from the wire end to the workpiece was very short (3-4 mm). In this case, the gaseous jet after impinging on the weld pool spread around and then went upward. Because of the influence of the water environment, the expansion of the bubbles in the horizontal direction was limited by the effect of water pressure, which resulted in the upward movement of gas flow. This can be clearly observed from the expansion behavior of bubbles, i.e., the bubbles expanded horizontally to a certain size and then moved directly upward. The bubble expansion and spatter behaviors under the action of gas flow showed that gas flow impact forces exist. 


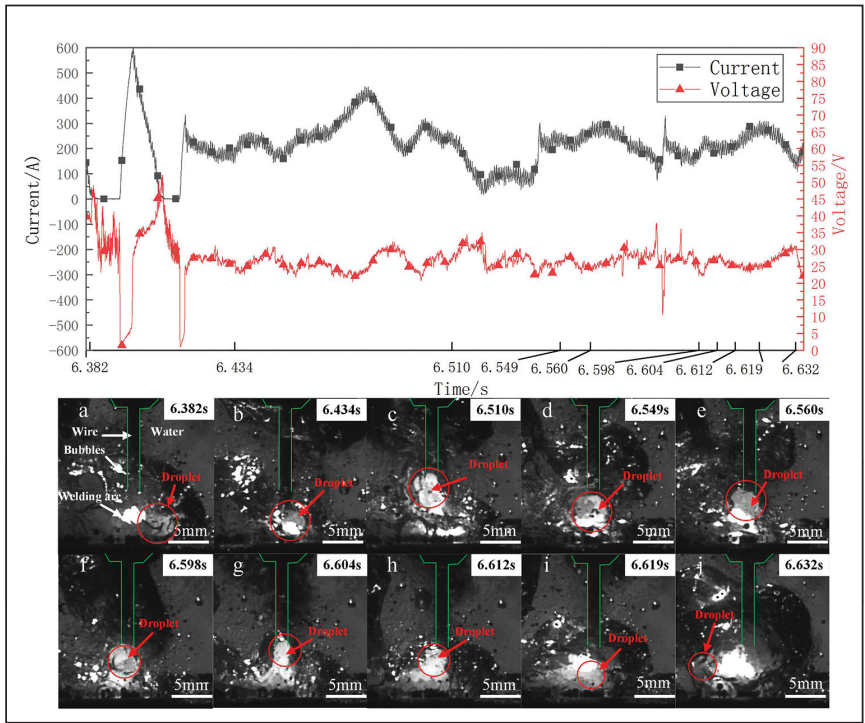

Fig. 8-Metal transfer process under the constant-voltage mode.

Therefore, when the new bubbles expanded horizontally, the acting forces applied on the droplets were not significant. With the welding process going on, the existence of the molten pool also heated the water to evaporate, increasing the gas production so that the equilibrium diameter of the bubbles expanded and the gas flow impact acting on the droplets in the bubbles was significantly reduced.

Under the adhesion detachment mode, as shown in Fig. $5 \mathrm{~B}$, the new bubbles were connected with the previous one when the bubbles separated from each other. The generated gas in the new bubbles flowed upward through the common gas channel between two bubbles and got into the rising, old bubble's space. The shape of the bubbles was cylinder or sleeve shaped. Bubbles tended to expand upward. The gas flow directions are shown using dotted lines in Fig. 5B.

The acting forces applied on the droplets in the bubbles were much greater than that under the completely detached mode. When new bubbles rose up, the volume of the bubble in the arc column region was small because of the necking of the former bubble. Meanwhile, the gas production inside new bubbles was still intense, and large amounts of gas rapidly expanded upward in a short time. The forces acting on the droplet mainly included drag force from the former bubble and impact force from the gas flow in the new bubble. A droplet that has grown to a certain volume can be easily pushed upward under the drag force to impact force. In this case, once the droplet detached, the upward momentum made it become spatter. As shown in Fig. 3B, spatter is formed along with the bubble separating process; it can be clearly observed at the junction of former and new bubbles.

To explore the effects of the above-mentioned, twobubble separation modes on metal transfer, statistical analysis was carried out. It was found that when the bubbles were separated completely, a certain drag force acted on the droplets.

The drag force made the droplets move upward along the wire, which resulted in the long residence time of the droplets in the deviated large droplet stage and the longer transfer time. However, the drag force was not big enough to push the

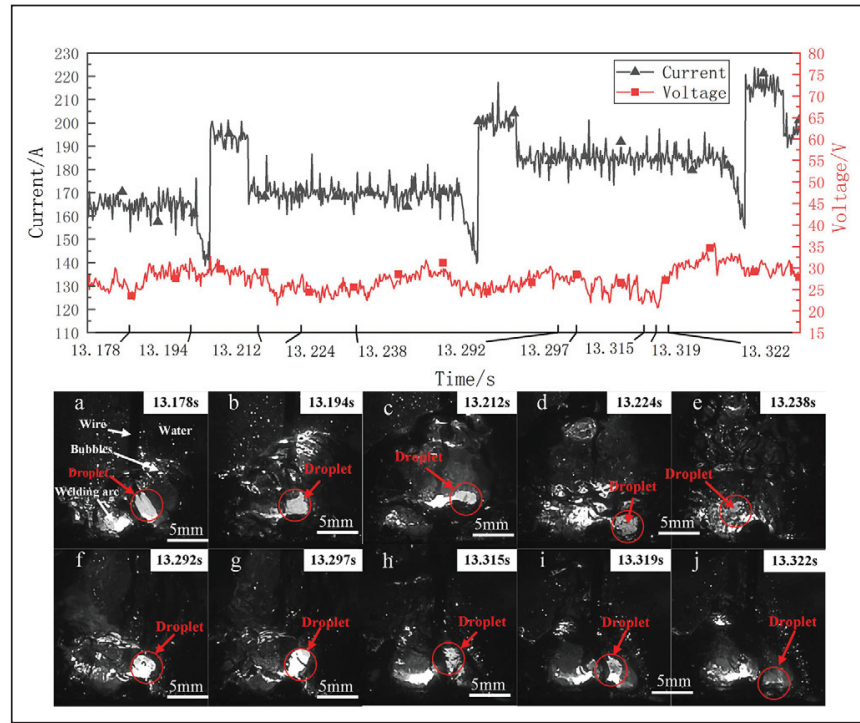

Fig. 9-Metal transfer process under the optimized pulsecurrent waveform mode.

droplet to detach from the wire end and form spatter.

As shown in Fig. 6, two typical periods of bubble detachment under the complete separation mode were selected, and the time of bubble necking was set as the starting time. Due to the interference of uneven light, to more easily identify the shape of the droplet, the droplet was marked with a circle and the outline of the torch was traced with a line. As seen in Fig. 6, because of the insufficient gas production, there were gaps between the former and new bubbles when the bubble necked and the surrounding water pressed the new bubble to form a shape like an inverted bowl.

As the welding process proceeded, the new bubble expanded horizontally while the former one moved upward. Generally, when the bubble necking occurs, the droplet is located at the upper position of the new bubble, as shown in Fig. $6 \mathrm{~A}$ and $\mathrm{F}$ at $0 \mathrm{~ms}$. At this point, the droplet was not only subjected to the drag force produced by the upward detachment of bubbles but also to the upward impact of the gas in the new bubbles below. However, due to the effect caused by the complete separation of bubbles (i.e., the relative reduction of gas production at this time), the upward gas impact effect in the new bubbles was not obvious. Therefore, with the separation of the former bubbles, the droplet tended to move upward with a small amplitude, as shown in Fig. 6C and $\mathrm{H}(9.71 \mathrm{~ms})$. Under the action of arc forces, surface tension, droplet gravity, etc., the droplet was not dragged away from the wire end by the drag force when the former bubble moved upward. Instead, it remained in a sagging status after rising for a limited distance. Because the former bubble moved away, there was only a very thin gas layer above the molten droplet. Inside the continuously growing and expanding new bubbles, the droplets were pulled back into the new bubbles, as shown in Fig. 6E and J (22.66 and $25.90 \mathrm{ms,}$ respectively). In the complete bubble separation mode, as shown in Fig. 6E and $\mathrm{H}$, there was no gas flow channel between the two bubbles, but they were completely separated.

As shown in Fig. 7, two typical periods of bubble separating behaviors under the adhesion separation mode were se- 


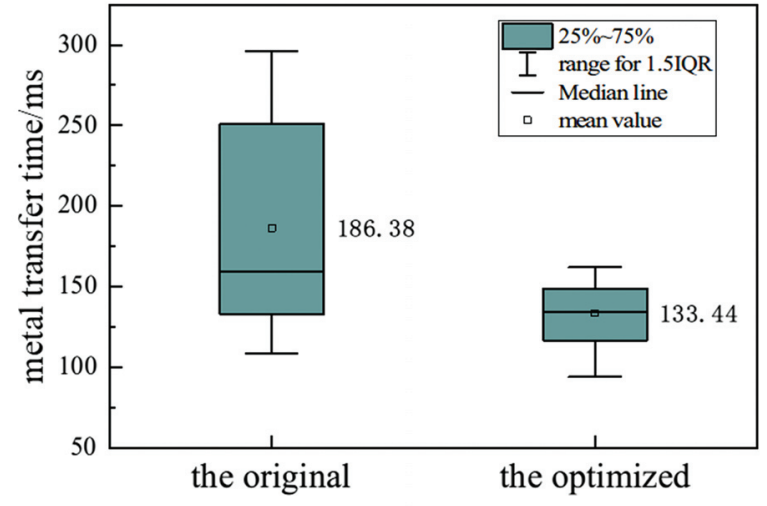

Fig. 10 - Metal transfer cycles under different pulse-current waveforms.

lected. The outline of the bubble was marked with lines. Similarly, the initial time (0 ms) was set as the bubble necking beginning. The bubbles' morphology was quite different from that under the complete detachment mode. In Fig. 7, it can be can seen that in the bubble adhesion separation mode, the bubble did not completely separate, but there was an obvious gas flow channel in the middle of the bubble. Theoretically, there was no water pressure difference between the former bubble and the new bubbles. The new bubbles had obvious, oblique boundaries rather than sphere shapes; the whole was like a cylinder or sleeve, as shown in Fig. 7D (29.14 ms). As previously analyzed, the droplets under this mode easily formed spatter directly with the former bubbles floating out of the arc column. The droplets were pushed away from the wire tip shortly after the bubble necking, as shown in Fig. 7B and G (9.71 and $3.24 \mathrm{~ms}$, respectively). Upward and outward movement of the droplets can be observed due to the initial momentum. A droplet even flew far away from the welding area and formed a spatter, as shown in Fig. 7E.

Based on the above analysis, it can be concluded that the impact forces acting on droplets reached the maximum when the bubble necked. At this moment, the gas production in the bubble determined the effect of the bubble influence acting on droplets, while the current value directly affected the gas production rate in the bubble. When the current increased, the bubbles were in the adhesion separation mode, and the droplets probably moved out to form spatter. When the current decreased, the bubble's evolution worked under the complete separation mode. In this case, with the significantly decreased bubble drag force and arc forces, the droplets detached from the welding wire and transferred to the molten pool more smoothly. Therefore, by reasonably adjusting the pulse-current waveform and its output frequency, the regulation and control of metal transfer was realized.

\section{Control Effect Evaluation of Optimized Pulse-Current Wet Welding}

In the underwater wet FCAW process, the constant-volt-

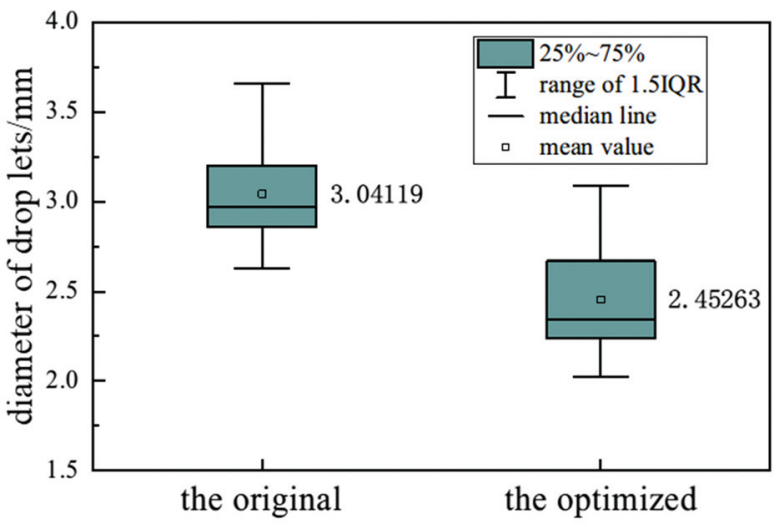

Fig. $11-$ Droplet diameters under different pulse-current waveforms.

age mode was used to keep the stable arc length. However, the periodically detaching bubbles strongly interfered with the arc burning and the droplet transfer processes, which led to poor weld formation. In part 1 of this two-part report, a novel pulse-current welding method was proposed, and experiments showed the pulse current had a better control effect on the droplet transfer and weld formation. In this case, by considering the bubble separation modes, the pulse-current waveform and its output frequency were optimized accordingly. By examining the control effect of optimized, pulse-current wet welding, the metal transfer processes and the improved bubble evolution behaviors were compared with both constant voltage and the original pulse-current FCAW.

First, welding experiments under constant voltage were carried out for comparison. Under the constant-voltage welding condition, the power source kept the arc voltage almost constant by adjusting the welding current from time to time to match the wire feed rate. As shown in Fig. 8, the metal transfer images and corresponding electrical signals under constant-voltage and pulse-current conditions were collected, compared, and analyzed. The images show a typical metal transfer cycle in the constant-voltage welding process with a period of $250 \mathrm{~ms}$. There was already an obvious deviation of the droplet at $6.549 \mathrm{~s}$ (Fig. 8D), $115 \mathrm{~ms}$ later than that of the initial droplet stage (Fig. 8A). Thereafter, the current decreased, and the droplet continued to grow under the action of the bubble drag force and arc forces. The welding current varied irregularly during this stage until the droplet grew to a sufficient size to contact the molten pool under gravity (Fig. 8G, 6.604 s). A short circuit occurred and the metal transferred to the molten pool due to the surface tension. The duration of the deviated large droplet stage was about $94 \mathrm{~ms}$.

In the process of metal transfer, the current decreased when the bubble necked, which also resulted in the formation of the bubble complete separation mode. In Fig. 8 (6.51 s), it can be clearly seen that there was an obvious drag force acting on the droplet while the bubble floated up and separated. Additionally, the droplet moved upward in an ellipsoid shape due to the pulling effect from the intense gas 


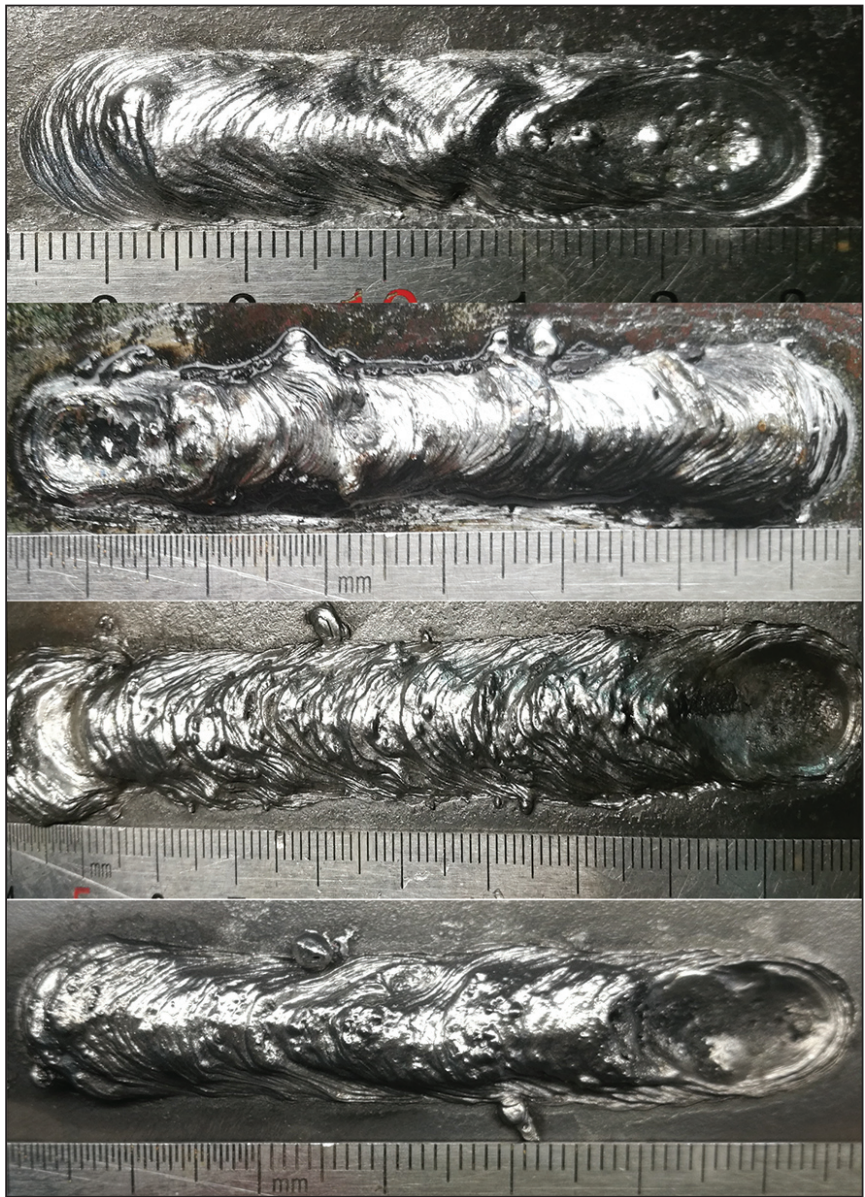

flow. Finally, the bubble completed the separation. Bubbles in the complete separation mode exerted a considerable drag force on the droplet, but the gas in the new bubble did not exert a great impact on the droplet. Therefore, the droplet did not fly out of the bubble to form spatter.

Second, as discussed in part 1 of this report, the original applied pulse current changed the period from 150 to 200 $\mathrm{ms}$ in the constant-voltage mode into 30 to $200 \mathrm{~ms}$. That is to say, the metal transfer period can be effectively shortened to some extent. During the mentioned six stages of metal transfer, the long duration of the deviated large droplet stage was the main factor causing the long period, low frequency, and instability of the metal transfer. The acquired results show that the pulse current in this stage significantly influenced the droplet separation behaviors and decreased the metal transfer cycle in a large amplitude. However, the effect of the present pulse current was not stable and accurate. The control effect needed to be further optimized. In this paper, the influences of the bubble separation mode were considered significant for the metal transfer processes. The optimized waveform was expected to more effectively regulate the metal transfer processes.

Third, the physical processes of the optimized pulsecurrent FCAW were visually investigated. Figure 9 shows two metal transfer cycles during pulse-current wet welding. Under the pulse-current mode and the parameters shown in Figs. 4 and 9A, a significant droplet deviation can be seen. Thereafter, the current experienced a steep rise after the decrease. After $34 \mathrm{~ms}$ (Fig. 9C, $13.212 \mathrm{~s}$ ), the droplet detached

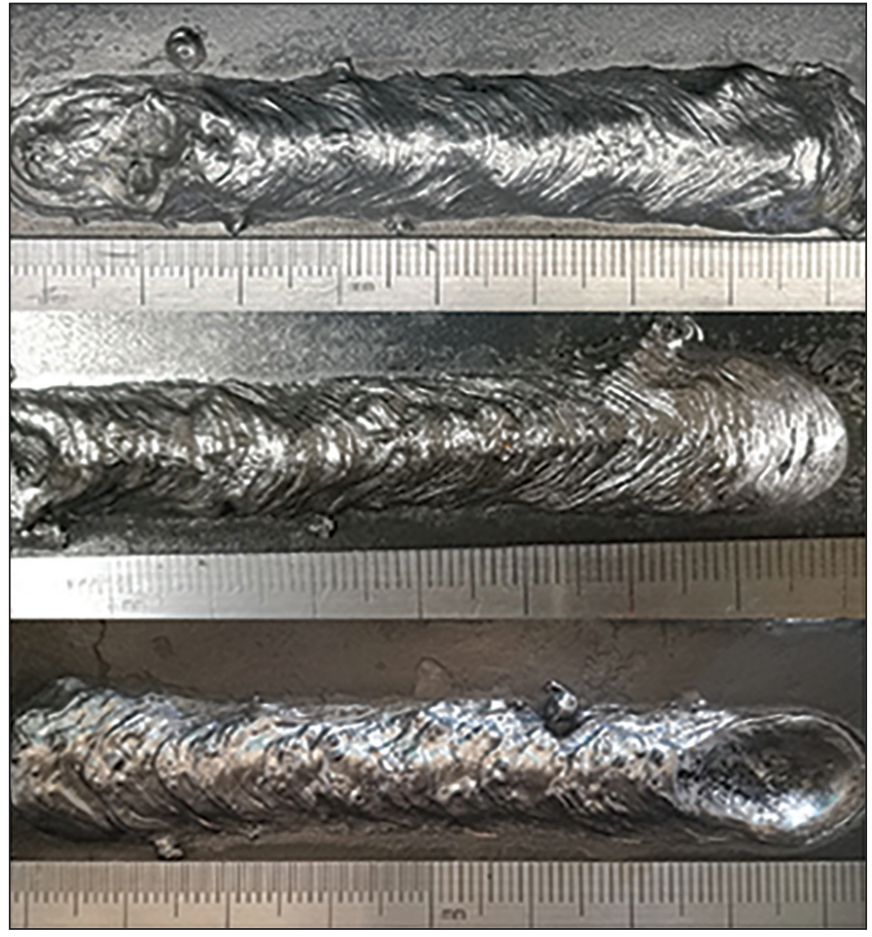

Fig. 12 - Comparison of weld bead appearance under the original waveform (left) and the optimized waveform (right).

from the tip of the wire toward one side of the welding wire and then dropped into the molten pool at $13.224 \mathrm{~s}$, as shown in Fig. 9D. When the droplet entered the molten pool, a new one was clearly observed after $14 \mathrm{~ms}$ at $13.238 \mathrm{~s}$ (Fig. 9E). After another $54 \mathrm{~ms}$, the new droplet grew to a certain size and then deviated obviously (Fig. 9F, 13.292 s). Subsequently, from Fig. $9 \mathrm{G}$ to J, the droplet went through short-deviation detaching and transferring stages. The droplet finally entered into the molten pool at $13.322 \mathrm{~s}$.

During the droplet transfer processes, the bubble evolution processes played an important role. At $13.238 \mathrm{~s}$, the bubble necking can be seen. With the decreased current in the pulse-current waveform, the gas production decreased to a certain extent, and the bubbles separated from each other under the complete separation mode. Compared with the metal transfer processes in constant-voltage welding, the droplet directly detached from the wire when it entered the deviated, large droplet stage under the applied pulse current with the new waveform. It greatly reduced the duration of the deviated large droplet stage, which is considered the main factor determining the long metal transfer cycles. The droplet transfer was significantly improved with smaller diameter and higher transfer frequency.

In the metal transfer process (from Fig. 9A to D), it can be clearly seen that no bubble separation occurred, and the shape of the bubble was more regular. Because the droplet and welding arc were always covered inside, the bubble didn't nearly influence the droplet behaviors during this period. The regular bubble shape shows that its expansion was uniform, and the gas flow did not fluctuate greatly.

Therefore, combined with the electrical signal waveform at this moment, what could be concluded is that the droplet was repelled outward and forced to detach from the wire end 
at $13.212 \mathrm{~s}$, as shown in Fig. 9C, under the action of optimized pulse current. Please note that the sharply increased welding current (between 13.194 and $13.212 \mathrm{~s}$ ) at the third stage of the new waveform, as shown in Fig. 4, produced gases. The inner intense gas flow was applied to the droplet and helped it to detach from the wire end. Due to the thermal lag effect and droplet movement time, the deviated and detached droplet was observed after the pulse-current application. In addition, the bubble necked near the workpiece, and then the droplet dropped into the molten pool at $13.224 \mathrm{~s}$.

The second metal transfer period was shown from Fig. 9E to J. In Fig. 9D, the transferred droplet caused the bubble's oscillation and collapse; thus, a new bubble emerged. Different from the previous metal transfer cycle, the should-beseparating bubble collapsed, which disrupted the original purpose of the optimized current waveform. According to the initial purpose, the new waveform was designed to accelerate the bubble separation and the droplet transfer processes via adjusting drag force inside the bubbles. However, the above phenomenon verifies that the droplet detachment may not be accomplished by only relying on pulse current. The collapse of bubbles resulted in the slow growth of new bubbles.

As shown in Fig. 9F, at $13.292 \mathrm{~s}$ the bubble was still small even after the current went through a sharp increase. At the following moment of $13.297 \mathrm{~s}$, the droplet volume seemed relatively larger than that at $13.212 \mathrm{~s}$ of the previous transfer period, as shown in Fig. 9C; the droplet was not directly repelled off the wire end compared with that at $13.212 \mathrm{~s}$. In addition, inside the small-volume bubble, as shown in Fig. 9G, the bottom of the droplet did not neck like that at $13.212 \mathrm{~s}$. Therefore, the droplet only oscillated during this period without detaching. After another $25 \mathrm{~ms}$ (Fig. 9J, $13.322 \mathrm{~s}$ ), the droplet dropped off under the action of inertia momentum and gravity, entering into the molten pool. Compared with the former metal transfer process, the droplet in this period experienced two current waveforms before detaching.

By analyzing both metal transfer images and electrical signals, it was found that the frequency of bubble evolution coincided with the frequency of the present optimized pulse-current waveform. That is to say, the optimized bubble evolution frequency was approximately $14 \mathrm{~Hz}$ with the period of about $71 \mathrm{~ms}$. While under the original pulsecurrent waveform (average current $190 \sim 200 \mathrm{~A}$ ), as shown in Fig. 2, the bubble evolution frequency was about $18 \sim 19$ $\mathrm{Hz}$. Therefore, it can be concluded that the optimized pulsecurrent waveform also significantly reduced the bubble evolution frequency, which was important for maintaining stable arc burning and metal transfer.

For the pulse-current waveform, the amplitude from the lowest value $(150 \mathrm{~A})$ to the pulse current $(210 \mathrm{~A})$ was set as 60 A. The time lengths of different waveform segments can be designed according to the periodical evolution and behaviors of the bubble and droplet. Droplets and bubbles grew steadily under a certain current. When the droplets deviated, the rapidly changed welding current caused the bubbles to neck and separate. Under the combined influence of pulse current and bubble behaviors, the droplets entered the deviated large droplet stage, and the detachment occurred soon after.

\section{Improved Metal Transfer and Weld Formation}

In the underwater wet welding process, a large droplet size and a low transfer frequency are the main reasons for poor weld formation. Based on analyzing the signals from Fig. 9, it was found that under the optimized pulse-current waveform, the droplet transfer process was more stable than that of the original one (Ref. 3). In other words, under the original pulse current, there existed a large portion of the adhesion separation mode as well as some of the complete separation mode in the bubble separation processes. On one side, the typical metal transfer under the adhesion separation mode was the repelled globular transfer with a large droplet size and a low transfer frequency. On the other side, under the optimized pulse-current waveform, a bigger portion of the complete separation modes and a more stable welding process were obtained, and the metal transfer mode still repelled globular transfer but with a smaller droplet size and a higher transfer frequency. Therefore, it regulated and controlled the metal transfer process to a certain extent. However, there were still some unexpected phenomena in the welding process, such as the above-mentioned bubble collapse due to droplets entering the molten pool. Considering these situations, further optimization of the waveform is needed in the future.

The improved droplet transfer process was conducive to obtaining good weld formation. The metal transfer cycles of the original waveform and the optimized waveform were statistically analyzed, as shown in Fig. 10. Compared with the metal transfer time of the original preset waveform, the variation range of metal transfer periods under the optimized waveform was significantly reduced, i.e., the metal transfer became more stable with less fluctuation caused by unpredictable disturbances. The distribution range of the metal transfer cycles became much narrower. Furthermore, the average transfer period significantly reduced from 186.38 to $133.44 \mathrm{~ms}$. The decreased metal transfer period means the increased metal transfer frequency can reach up to $7.52 \mathrm{~Hz}$ under this optimized waveform. That is to say, the metal transfer processes became more stable.

The droplet diameters were also critical to the stability of the metal transfer processes. The droplet diameters under the original and optimized waveforms were calculated and compared. As shown in Fig. 11, the average droplet diameter under the optimized waveform decreased to $2.45 \mathrm{~mm}$ from the former $3.04 \mathrm{~mm}$ under the original waveform. The maximum droplet diameter did not exceed twice the diameter of the wire, and the minimum was close to the wire diameter of $1.6 \mathrm{~mm}$. The reduction of the droplet diameter was beneficial to the stability of the welding process to a certain extent. In other words, the optimized metal transfer processes had a higher frequency and smaller diameters. Thus, the impact from the liquid droplets on the weld pool can be significantly reduced along with less spatter generation.

As mentioned in part 1 of this two-part report, the novel pulse-current underwater FCAW was better than the constant voltage mode in improving weld formation (Ref. 3). In this section, we further compared the weld formation under the original waveform and the optimized waveform. As shown in Fig. 12, the weld bead formation was further improved by the optimized pulse current. The weld bead sur- 
face became more uniform with less defects, such as spatter, weld beading, etc. Different from the images on the left, the weld beads on the right had nearly constant widths and reinforcement. It can be deduced that, under the optimized pulse-current FCAW, the molten metal droplets were much more smoothly transferred to the weld pool and then solidified with uniform weld beads formed. Compared to the serious spatter generation in traditional underwater FCAW, much less droplets were transformed into spatter (Ref. 6).

\section{Conclusions}

1) During pulse-current wet FCAW, the periodically changed current produced two different bubble separation modes, i.e., complete separation and adhesion separation. Different bubble shapes were observed due to the complex inner gas expansion behaviors. When a new bubble was formed under the adhesion separation mode, there was a junction gas channel between the former bubble and the new bubbles. The gas flow inside the channel significantly influenced the welding processes.

2) According to the synchronous observation of bubble and droplet behaviors, it was found that two bubble separation modes had different influences on the droplet transfer. Under the adhesion separation mode, droplets were subject to not only the gas flow impact inside the new bubbles, but also the drag force produced by the intense gas flow inside the junction channel between the two bubbles. The resultant forces resisted the droplets falling downward and even pushed them out of the bubbles. The resistance force applied on droplets was relatively small in the complete separation mode, which was more conducive for the more smooth droplet transfer.

3) When optimizing the pulse-current waveform, the pulsecurrent influences on bubbles were taken into account for significantly shortening the deviated large droplet stage of metal transfer. Four sections of the new waveform were defined for droplet growing, fluctuation, detaching, and transferring, respectively. Note the current was rapidly decreased in the second section to reduce not only the repulsive force on the droplet but also to decrease the generated gas flow to avoid the bubble adhesion separation. With the optimized waveform, the bubble expansion and evolution happened in the complete separation mode. The quickly and completely separated bubbles could avoid the intense gas flow disturbances on the detaching droplet, and spatter was not formed.

4) Under the optimized pulse-current waveform, the droplet transfer process was more stable than that of the original one. It can effectively regulate and control the metal transfer as well as the bubble evolution processes. The metal transfer cycle range became narrower, the average transfer cycle was reduced by $28.5 \%$, the droplet diameter was reduced by $19 \%$, and metal transfer frequency reached 7.52 $\mathrm{Hz}$. At the same time, the optimized pulse waveform significantly improved weld formation with less spatter and uniform appearance. Note that the complicated underwater environment may disrupt the process unexpectedly and occasionally, such as a sudden bubble collapse due to droplets entering the molten pool. The robustness of this study could be enhanced in the future.

\section{Acknowledgments}

The authors are grateful to the National Natural Science Foundation of China (Grant No. 51675310) and the Fundamental Research Funds of Shandong University (No. 2015TB002) for their financial support of this project.

\section{References}

1. Jia, C. B., Zhang, Y., Zhao, B., et al. 2016. Visual sensing of the physical process during underwater wet FCAW. Welding Journal 95(6): 202-s to 209-s.

2. Guo, N., Fu, Y., Feng, J., et al. 2016. Classification of metal transfer mode in underwater wet welding. Welding Journal 95(4): 133 -s to 140 -s.

3. Chuanbao, J., Wu, J., Han, Y., et al. 2020. Underwater pulsecurrent FCAW - Part 1: Waveform and process features. Welding Journal 99(5): 135-s to 145-s. DOI: 10.29391/2020.99.013

4. Tsai, C.-L., and Masubuchi, K. 1979. Mechanisms of rapid cooling in underwater welding. Applied Ocean Research 1(2): 99-110. DOI: 10.1016/0141-1187(79)90023-3

5. Jia, C., Zhang, Y., Wu, J., et al. 2019. Comprehensive analysis of spatter loss in wet FCAW considering interactions of bubbles, droplets and arc - Part 2: Visualization \& mechanisms. Journal of Manufacturing Processes 40: 105-112. DOI: 10.1016/j.jmapro. 2019.03.011

6. Jia, C., Zhang, Y., Wu, J., et al. 2019. Comprehensive analysis of spatter loss in wet FCAW considering interactions of bubbles, droplets and arc - Part 1: Measurement and improvement. Journal of Manufacturing Processes 40: 122-127. DOI: 10.1016/j. jmapro.2019.03.013

JUNFEI WU, YANFEI HAN, CHUANBAO JIA (jiachuanbao@ sdu.edu.cn), QINGYUAN YANG, and CHUANSONG WU are with the Ministry of Education Key Laboratory for Liquid-Solid Structural Evolution and Processing of Materials, and the Institute of Materials Joining, Shandong University, Jinan, China. 